\title{
ERGODIC BEHAVIOUR OF EXTREME VALUES
}

\author{
S. CHENG, L. PENG and Y. QI
}

(Received 8 April 1998; revised 28 June 1999)

Communicated by V. Stefanov

\begin{abstract}
Let $\left\{X_{n}, n \geq 1\right\}$ be independent identically distributed random variables with a common non-degenerate distribution function $F$. For each $n \geq 1$, denote $M_{n}=\max \left\{X_{1}, \ldots, X_{n}\right\}$. Under certain conditions on $F$, there exist constants $a_{n}>0$ and $b_{n} \in \mathbb{R}$ such that $\left(M_{n}-b_{n}\right) / a_{n} \stackrel{d}{\rightarrow} G$. In this paper, we shall show that $\left\{\left(M_{n}-b_{n}\right) / a_{n}\right\}$ exhibits ergodic behaviour under additional conditions on $F$.

1991 Mathematics subject classification (Amer. Math. Soc.): primary 60F05.

Keywords and phrases: ergodic behaviour, extreme values, logarithmic means, almost sure convergence.
\end{abstract}

\section{Introduction}

Let $\left\{X_{n}, n \geq 1\right\}$ be a sequence of independent identically distributed random variables with $\mathbb{E} X_{1}=0$ and $\mathbb{E} X_{1}^{2}=1$. For each $n \geq 1$, set $S_{n}=\sum_{i=1}^{n} X_{i}$. Then for $x \in \mathbb{R}$

$$
\lim _{n \rightarrow \infty} \frac{1}{\log n} \sum_{k=1}^{n} \frac{1}{k} \mathbf{1}\left(\frac{S_{k}}{\sqrt{k}} \leq x\right)=\frac{1}{\sqrt{2 \pi}} \int_{-\infty}^{x} e^{-t^{2} / 2} d t \quad \text { almost surely. }
$$

This is known as the almost sure central limit theorem, and was proved first by Brosamler [1] and Schatte [8]. Recently, the related problems have attracted much attention (see Lacey and Philipp [6], Schatte [9-11]).

It was shown in Cheng et al. [2] that the above phenomenon holds also for extreme values (see Lemma 2 in Section 2). In this paper we consider the general problem related to maxima (see below).

We assume throughout that $\left\{X_{n}, n \geq 1\right\}$ are a sequence of independent identically distributed random variables with a common non-degenerate distribution function $F$.

(C) 2000 Australian Mathematical Society $0263-6115 / 2000 \$ A 2.00+0.00$ 
For each $n \geq 1$, denote

$$
M_{n}=\max \left\{X_{1}, \ldots, X_{n}\right\} .
$$

Suppose there exist constants $a_{n}>0$ and $b_{n} \in \mathbb{R}$ such that

$$
\left(M_{n}-b_{n}\right) / a_{n} \stackrel{d}{\rightarrow} G
$$

where $G$ is a non-degenerate distribution function. Then we say $G$ is an extreme value distribution and $F$ is in the domain of attraction of $G$ (notation: $F \in D(G)$ ).

It is well known that $G$ must be one of the following three types:

$$
\begin{aligned}
& G(x)=\Phi_{\alpha}(x)= \begin{cases}\exp \left\{-x^{-\alpha}\right\} & \text { if } x \geq 0, \\
0 & \text { if } x<0 ;\end{cases} \\
& G(x)=\Psi_{\alpha}(x)= \begin{cases}1 & \text { if } x \geq 0, \\
\exp \left\{-(-x)^{\alpha}\right\} & \text { if } x<0 ;\end{cases} \\
& G(x)=\Lambda(x)=\exp \left\{-e^{-x}\right\} \text { for } x \in \mathbb{R},
\end{aligned}
$$

where $\alpha>0$ (see [7, Proposition 0.3]). Furthermore $a_{n}$ and $b_{n}$ can be chosen as

$$
a_{n}= \begin{cases}U(n) & \text { if } G(x)=\Phi_{\alpha}(x), \\ x_{F}-U(n) & \text { if } G(x)=\Psi_{\alpha}(x), \\ U(n e)-U(n) & \text { if } G(x)=\Lambda(x),\end{cases}
$$

and

$$
b_{n}= \begin{cases}0 & \text { if } G(x)=\Phi_{\alpha}(x), \\ x_{F} & \text { if } G(x)=\Psi_{\alpha}(x), \\ U(n) & \text { if } G(x)=\Lambda(x),\end{cases}
$$

where $x_{F}:=\sup \{x: F(x)<1\}$ and $U(x):=\inf \{y: 1 /(1-F(y))>x\}$.

It is important to have necessary and sufficient conditions for a distribution to belong to the domain of attraction of an extreme value distribution. Some characterisation theorems can be found in [7, Chapter 1]. For example, $F \in D\left(\Phi_{\alpha}\right)$ if and only if

$$
\lim _{t \rightarrow \infty} \frac{U(t x)}{U(t)}=x^{1 / \alpha} \quad \text { for all } x>0
$$

(notation: $U \in R V_{1 / \alpha}$ ).

Assume that (1.1) holds. If there exists a positive sequence $\left\{r_{n}, n \geq 1\right\}$ with $\sum_{k=1}^{\infty} r_{k}=\infty$ such that

$$
\lim _{n \rightarrow \infty} \frac{1}{\sum_{k=1}^{n} r_{k}} \sum_{k=1}^{n} r_{k} f\left(\frac{M_{k}-b_{k}}{a_{k}}\right)=\int_{\mathbf{D}} f(x) G(d x) \text { almost surely }
$$


(with $\mathbf{D}:=\{x: 0<G(x)<1\}$ ) holds for a class of functions $f$, then we may say that the sequence $\left\{\left(M_{n}-b_{n}\right) / a_{n}\right\}$ has ergodic behaviour. It is natural to consider the case where $r_{n}=n^{-\gamma}$ with $0<\gamma \leq 1$. Unfortunately, for $\gamma \in(0,1)$, the above equation is not true even for the indicator function (see Cheng et al. [2]). Hence we only consider the case $\gamma=1$, that is the logarithmic means. In the present paper, the following results are obtained (proofs are given in Section 2).

THEOREM 1. Suppose (1.1) holds for $G=\Phi_{\alpha}, F(0-)=0$, and $a_{n}$ and $b_{n}$ are defined by (1.2) and (1.3). Assume $f$ is an almost everywhere continuous function which is defined on $(0, \infty)$. If there are constants $B>0, \beta \in(0, \alpha)$ and $\tau>0$ such that

$$
|f(x)| \leq B\left(x^{\beta}+x^{-\tau}\right) \quad \text { for all } x>0,
$$

then

$$
\lim _{N \rightarrow \infty} \frac{1}{\log N} \sum_{n=1}^{N} \frac{1}{n} f\left(\frac{M_{n}-b_{n}}{a_{n}}\right)=\int_{0}^{\infty} f(x) \Phi_{\alpha}(d x) \text { almost surely. }
$$

REMARK 1. Condition (1.4) ensures that $\left|\int_{0}^{\infty} f(x) \Phi_{\alpha}(d x)\right|<\infty$. Indeed, (1.5) still holds if we replace (1.4) by assuming that there are constants $\beta \in(0, \alpha)$ and $\tau \in(0,1 / \alpha)$ such that

$$
|f(x)| \leq B\left(x^{\beta}+e^{x^{-\tau}}\right) \quad \text { for all } x>0 .
$$

REMARK 2. Assume (1.1) holds for $G=\Phi_{\alpha}$, and $f$ is an almost sure continuous function $f$ which is defined on $(-\infty, \infty)$. If there are constants $B>0$ and $\beta \in(0, \alpha)$ such that

$$
|f(x)| \leq B(|x|+1)^{\beta} \quad \text { for } x \in \mathbb{R},
$$

then (1.5) holds.

Note that since

$$
\int_{0}^{\infty} x^{\beta} \Phi_{\alpha}(d x)=\Gamma(1-\beta / \alpha)
$$

we have

$$
\lim _{N \rightarrow \infty} \frac{1}{\log N} \sum_{n=1}^{N} \frac{M_{n}^{\beta}}{n a_{n}^{\beta}}=\Gamma(1-\beta / \alpha) \quad \text { almost surely. }
$$


THEOREM 2. Suppose (1.1) holds for $G=\Psi_{\alpha}$, and $a_{n}$ and $b_{n}$ are defined by (1.2) and (1.3). Assume $g$ is an almost everywhere continuous function which is defined on $(-\infty, 0)$. If there are constants $B>0, \beta \in(0, \alpha)$ and $\tau>0$ such that

$$
|g(x)| \leq B\left(|x|^{-\beta}+|x|^{\tau}\right) \quad \text { for all } x<0,
$$

then

$$
\lim _{N \rightarrow \infty} \frac{1}{\log N} \sum_{n=1}^{N} \frac{1}{n} g\left(\frac{M_{n}-b_{n}}{a_{n}}\right)=\int_{-\infty}^{0} g(x) \Psi_{\alpha}(d x) \quad \text { almost surely. }
$$

REMARK 3. Assume (1.1) holds for $G=\Psi_{\alpha}$, and $g$ is an almost everywhere continuous function which is defined on $(-\infty, \infty)$. If there are constants $B>0$ and $\beta>0$ such that

$$
|g(x)| \leq B(|x|+1)^{\beta} \text { for } x \in \mathbb{R},
$$

then (1.8) holds.

Note that since for any positive integer $\beta$

$$
\int_{-\infty}^{0} x^{\beta} \Psi_{\alpha}(d x)=(-1)^{\beta} \Gamma(1+\beta / \alpha)
$$

we have

$$
\lim _{N \rightarrow \infty} \frac{1}{\log N} \sum_{n=1}^{N} \frac{\left(M_{n}-x_{F}\right)^{\beta}}{n a_{n}^{\beta}}=(-1)^{\beta} \Gamma(1+\beta / \alpha) \quad \text { almost surely. }
$$

THEOREM 3. Suppose (1.1) holds for $G=\Lambda$, and $a_{n}$ and $b_{n}$ are defined by (1.2) and (1.3). Assume $h$ is an almost everywhere continuous function which is defined on $(-\infty, \infty)$. If there are constants $B>0$ and $\beta>0$ such that

$$
|h(x)| \leq B(|x|+1)^{\beta} \quad \text { for } x \in \mathbb{R},
$$

then

$$
\lim _{N \rightarrow \infty} \frac{1}{\log N} \sum_{n=1}^{N} \frac{1}{n} h\left(\frac{M_{n}-b_{n}}{a_{n}}\right)=\int_{-\infty}^{\infty} h(x) \Lambda(d x) \text { almost surely. }
$$

REMARK 4. Under the conditions of Theorem 3, if $\beta$ is a positive integer, then

$$
\lim _{N \rightarrow \infty} \frac{1}{\log N} \sum_{n=1}^{N} \frac{\left(M_{n}-b_{n}\right)^{\beta}}{n a_{n}^{\beta}}=(-1)^{\beta} \Gamma^{(\beta)}(1) \quad \text { almost surely, }
$$

where $\Gamma^{(\beta)}(1)$ denotes the $\beta$-th derivative of the gamma function at $x=1$. 
REMARK 5. According to [7, Proposition 2.1], if (1.1) holds, then under additional conditions on the left tail of $F$, we have

$$
\lim _{n \rightarrow \infty} \mathbb{E}\left(\frac{M_{n}-b_{n}}{a_{n}}\right)^{\beta}= \begin{cases}\Gamma(1-\beta / \alpha) & \text { if } G(x)=\Phi_{\alpha}(x), \\ (-1)^{\beta} \Gamma(1+\beta / \alpha) & \text { if } G(x)=\Psi_{\alpha}(x), \\ (-1)^{\beta} \Gamma^{(\beta)}(1) & \text { if } G(x)=\Lambda(x),\end{cases}
$$

where $a_{n}$ and $b_{n}$ are defined by (1.2) and (1.3), and in the last two equations, $\beta$ should be positive integer. Thus by (1.6), (1.9) and (1.12) we have

$$
\lim _{N \rightarrow \infty} \frac{1}{\log N} \sum_{n=1}^{N} \frac{\left(M_{n}-b_{n}\right)^{\beta}}{n \mathbb{E}\left(M_{n}-b_{n}\right)^{\beta}}=1 \quad \text { almost surely. }
$$

REMARK 6. It is obvious that (1.1) holds for constants $a_{n}^{\prime}$ and $b_{n}^{\prime}$ which satisfy

$$
\left\{\begin{array}{l}
a_{n}^{\prime} / a_{n} \rightarrow 1 \\
\left(b_{n}^{\prime}-b_{n}\right) / a_{n} \rightarrow 0
\end{array}\right.
$$

as $n \rightarrow \infty$, where $a_{n}$ and $b_{n}$ are defined by (1.2) and (1.3) (see [7, Proposition 0.2]). Moreover, Remark 2, Remark 3 and Theorem 3 hold for above constants $a_{n}^{\prime}$ and $b_{n}^{\prime}$.

\section{Proofs}

For every measurable function $l$ let

$$
\mathbf{S}(l)=\{x: l \text { is continuous at } x\} .
$$

The proofs of our theorems are mainly based on the following lemmas. The proof of Lemma 1 below is very standard and we omit it.

LeMMA 1. Assume $\left\{Z, Z_{n}, n \geq 1\right\}$ is a sequence of random variables with distribution functions $\left\{G, G_{n}, n \geq 1\right\}$. Assume $\left\{Z_{n}\right\}$ converges in distribution to $Z$, that is

$$
\lim _{n \rightarrow \infty} G_{n}(x)=G(x), \quad \text { for } x \in \mathbf{S}(G) .
$$

If $l$ is a real-valued almost everywhere continuous function with respect to $G$, that is $\operatorname{Pr}(Z \in \mathbf{S}(l))=1$ and $\left\{l(Z), l\left(Z_{n}\right), n \geq 1\right\}$ is uniformly integrable (for definition of uniformly integrable, see [3, page 93]), then

$$
\lim _{n \rightarrow \infty} \mathbb{E} l\left(Z_{n}\right)=\mathbb{E} l(Z) .
$$


LEMMA 2. Assume (1.1) holds. Then

$$
\operatorname{Pr}\left\{\lim _{n \rightarrow \infty} \sup _{\mathbf{D}}\left|\frac{1}{\log n} \sum_{k=1}^{n} \frac{1}{k} \mathbf{1}\left(\frac{M_{k}-b_{k}}{a_{k}} \leq x\right)-G(x)\right|=0\right\}=1
$$

where $1(A)$ denotes the indicator function of set $A$, and $a_{n}$ and $b_{n}$ are defined by (1.2) and (1.3).

ProOF. See Cheng et al. [2].

Next we are going to prove our theorems. Set

$$
\Omega_{1}=\left\{\omega: \lim _{N \rightarrow \infty} \sup _{x \in \mathbf{D}}\left|\frac{1}{\log N} \sum_{n=1}^{N} \frac{1}{n} \mathbf{1}_{(-\infty, x]}\left(\frac{M_{n}-b_{n}}{a_{n}}\right)-G(x)\right|=0\right\} .
$$

From Lemma 2 we know

$$
\operatorname{Pr}\left(\Omega_{1}\right)=1
$$

Assume $\left\{W_{j}, j \geq 1\right\}$ is a sequence of independent random variables with common distribution function $\Phi_{1}$. It is easily seen that $\left\{U\left(1 /\left(1-\Phi_{1}\left(W_{j}\right)\right)\right), j \geq 1\right\}$ is a sequence of independent random variables which have the same distributions as $\left\{X_{j}, j \geq 1\right\}$. For the sake of simplicity, we assume that $X_{j}=U\left(1 /\left(1-\Phi_{1}\left(W_{j}\right)\right)\right)$, for $j \geq 1$. Using the well-known inequalities for regular variation and $\Pi$-variation (see Geluk and de Haan [4, Proposition 1.7.5 and Proposition 1.19.4]), we may concentrate on dealing with $\left\{W_{j}, j \geq 1\right\}$ (see (2.9) and (2.13) below).

For $1 \leq m \leq n$, set $W(n, m)=\max _{n-m+1 \leq j \leq n} W_{j}$. Obviously, $W_{n, m} / m$ has distribution function $\Phi_{1}$, and $M_{n}=U\left(1 /\left(1-\Phi_{1}(W(n, n))\right)\right)$ for $n \geq 1$. We also have

$$
W(n, n) \rightarrow \infty \quad \text { almost surely as } n \rightarrow \infty \text {. }
$$

ProOF (of Theorem 1). Put $\delta=(\beta / \alpha+1) / 2$ and $d^{2}=(\alpha+\beta) /(2 \beta)$. Then $d>1$ and $\delta \in(0,1)$. Throughout the proof we use $C$ to denote a positive constant, and we let $O(1)$ refer to almost surely.

We write

$$
\Omega_{2}=\left\{\omega: \limsup _{N \rightarrow \infty} \frac{1}{\log N} \sum_{n=1}^{N} \frac{1}{n}\left|f\left(\frac{M_{n}}{a_{n}}\right)\right|^{d}<\infty\right\} .
$$

First we show that

$$
\operatorname{Pr}\left(\Omega_{2}\right)=1
$$


Write $S_{N}=(\log N)^{-1} \sum_{n=1}^{N} n^{-1}\left|f\left(M_{n} / a_{n}\right)\right|^{d}$. Then (1.4) implies

$$
\begin{aligned}
S_{N} \leq & C\left(\frac{1}{\log N} \sum_{n=1}^{N} \frac{1}{n}\left(\frac{M_{n}}{a_{n}}\right)^{-d \tau}+\frac{1}{\log N} \sum_{n=1}^{N} \frac{1}{n}\left(\frac{M_{n}}{a_{n}}\right)^{d \beta}\right) \\
= & C\left(\frac{1}{\log N} \sum_{n=1}^{N} \frac{1}{n}\left(\frac{U\left(1 /\left(1-\Phi_{1}(W(n, n))\right)\right)}{U(n)}\right)^{-d \tau}\right. \\
& \left.+\frac{1}{\log N} \sum_{n=1}^{N} \frac{1}{n}\left(\frac{U\left(1 /\left(1-\Phi_{1}(W(n, n))\right)\right)}{U(n)}\right)^{d \beta}\right) \\
:= & C\left(S_{N}^{(1)}+S_{N}^{(2)}\right) .
\end{aligned}
$$

Since $U \in R V_{1 / \alpha}$, Potter-bound inequality (see Geluk and de Haan [4, Proposition 1.7.5]) implies that there exists $t_{0}>0$ such that

$$
\frac{U(t x)}{U(t)} \leq 2 x^{d / \alpha}
$$

for all $t>t_{0}$ and $x \geq 1$. Since $U(x)$ is non-decreasing, we have

$$
\frac{U\left(1 /\left(1-\Phi_{1}(W(n, n))\right)\right)}{U(n)} \leq 1+\frac{2}{\left(n\left(1-\Phi_{1}(W(n, n))\right)\right)^{d / \alpha}}
$$

for all $n \geq t_{0}$. Hence

$$
\begin{aligned}
S_{N}^{(2)} & =O(1)+C \frac{1}{\log N} \sum_{n=1}^{N} \frac{1}{n}\left(n\left(1-\Phi_{1}(W(n, n))\right)\right)^{-d^{2} \beta / \alpha} \\
& =O(1)+C \frac{1}{\log N} \sum_{n=1}^{N} \frac{1}{n}\left(n\left(1-\Phi_{1}(W(n, n))\right)\right)^{-\delta} \\
& =O(1)+\frac{O(1)}{\log N} \sum_{n=1}^{N} \frac{1}{n}\left(\frac{W(n, n)}{n}\right)^{\delta}
\end{aligned}
$$

since $1-\Phi_{1}(W(n, n)) \sim(W(n, n))^{-1}$ holds almost surely from (2.6).

Note that for each $N \geq 2$, there exists $m \geq 2$ such that $2^{m-1} \leq N<2^{m}$, and

$$
\begin{aligned}
\frac{1}{\log N} & \sum_{n=1}^{N} \frac{1}{n}\left(\frac{W(n, n)}{n}\right)^{\delta} \\
& \leq \frac{1}{(m-1) \log 2} \sum_{n=1}^{2^{m}} \frac{1}{n}\left(\frac{W(n, n)}{n}\right)^{\delta} \\
& \leq \frac{1}{(m-1) \log 2} \sum_{j=1}^{m} \sum_{n=2^{j-1}}^{2^{j}} \frac{1}{n}\left(\frac{W(n, n)}{n}\right)^{\delta}
\end{aligned}
$$




$$
\begin{aligned}
& \leq \frac{2^{\delta+1}}{m-1} \sum_{j=1}^{m}\left(\frac{W\left(2^{j}, 2^{j}\right)}{2^{j}}\right)^{\delta} \\
& \leq \frac{2^{\delta+1}}{m-1} \sum_{j=1}^{m} \frac{1}{2^{j \delta}}\left(\sum_{i=1}^{j}\left(W\left(2^{i}, 2^{i-1}\right)\right)^{\delta}+(W(1,1))^{\delta}\right) \\
& =\frac{2^{\delta+1}}{m-1}\left(\sum_{i=1}^{m}\left(W\left(2^{i}, 2^{i-1}\right)^{\delta}+(W(1,1))^{\delta}\right) \sum_{j=i}^{m} \frac{1}{2^{j \delta}}\right. \\
& \leq \frac{2^{\delta}}{2^{\delta}-1} \frac{1}{m-1}\left(\sum_{i=1}^{m}\left(\frac{W\left(2^{i}, 2^{i-1}\right)}{2^{i-1}}\right)^{\delta}+(W(1,1))^{\delta}\right) .
\end{aligned}
$$

Since $\left\{\left(W\left(2^{i}, 2^{i-1}\right) 2^{1-i}\right)^{\delta}, i \geq 1\right\}$ is a sequence of identical and independent random variables with finite means $\mathbb{E} W_{1}^{\delta}$, by the strong law of large numbers we have

$$
\frac{1}{m-1} \sum_{i=1}^{m}\left(\frac{W\left(2^{i}, 2^{i-1}\right)}{2^{i-1}}\right)^{\delta} \rightarrow \mathbb{E} W_{1}^{\delta} \quad \text { almost surely. }
$$

Therefore, by (2.8), (2.9) and (2.10) we have $S_{N}^{(2)}=O(1)$. In order to prove (2.7), we only need to show that

$$
S_{N}^{(1)}=O(1)
$$

Using Potter-bound inequality, for some $t_{1}>0$ and $C>0$

$$
\frac{U(t x)}{U(t)} \geq C x^{d / \alpha}
$$

holds for all $t>t_{1}, t x>t_{1}$ and $x \leq 1$. From (2.6), $1-\Phi_{1}(W(n, n)) \rightarrow 0$ almost surely. Hence,

$$
\operatorname{Pr}\left(1-\Phi_{1}(W(n, n)) \geq \frac{1}{t_{1}}, \text { infinitely often }\right)=0 .
$$

It is easy to check from (2.12) that

$$
\begin{aligned}
S_{N}^{(1)}= & \frac{1}{\log N} \sum_{n=1}^{N} \frac{1}{n}\left(\frac{U\left(1 /\left(1-\Phi_{1}(W(n, n))\right)\right)}{U(n)}\right)^{-d \tau} 1\left(\frac{1}{1-\Phi_{1}(W(n, n))} \leq t_{1}\right) \\
& +\frac{1}{\log N} \sum_{n=1}^{N} \frac{1}{n}\left(\frac{U\left(1 /\left(1-\Phi_{1}(W(n, n))\right)\right)}{U(n)}\right)^{-d \tau} 1\left(\frac{1}{1-\Phi_{1}(W(n, n))}>t_{1}\right) \\
= & O(1)+\frac{1}{\log N} \sum_{n=1}^{N} \frac{1}{n}\left(n\left(1-\Phi_{1}(W(n, n))\right)^{d^{2} \tau / \alpha}\right.
\end{aligned}
$$




$$
=O(1)+\frac{O(1)}{\log N} \sum_{n=1}^{N} \frac{1}{n}\left(\frac{n}{W(n, n)}\right)^{d^{2} \tau / \alpha} .
$$

For $N \geq 2,2^{m-1} \leq N<2^{m}$, it may easily be proved that

$$
\frac{1}{\log N} \sum_{n=1}^{N} \frac{1}{n}\left(\frac{n}{W(n, n)}\right)^{d^{2} \tau / \alpha} \leq \frac{C}{m-1} \sum_{j=1}^{m}\left(\frac{2^{j-1}}{W\left(2^{j}, 2^{j-1}\right)}\right)^{d^{2} \tau / \alpha},
$$

which is bounded almost surely by the strong law of large numbers since $\left\{(n / W(n, n))^{d^{2} \tau / \alpha}\right\}$ is a sequence of identical and independent random variables with finite means $\mathbb{E}\left(W_{1}\right)^{-d^{2} \tau / \alpha}$. Thus, $(2.11)$ is proved. This completes the proof of (2.7).

Set $\Omega=\Omega_{1} \cap \Omega_{2}$. From (2.5) and (2.7) we have $\operatorname{Pr}(\Omega)=1$. Put $K(N)=\sum_{n=1}^{N} 1 / n$. Fix $\omega \in \Omega$ and write

$$
F_{N}(x)=\frac{1}{K(N)} \sum_{n=1}^{N} \frac{1}{n} \mathbf{1}_{(-\infty, x]}\left(\frac{M_{n}}{a_{n}}\right), \quad x \in \mathbb{R} .
$$

Then $\left\{F_{N}\right\}$ is a sequence of distribution functions. Let $Z_{N}$ have distribution $F_{N}$ and $Z$ has distribution $\Phi_{\alpha}$. Since $K(N) / \log N \rightarrow 1$ as $N \rightarrow \infty$, we have

$$
\lim _{N \rightarrow \infty} \sup _{x}\left|F_{N}(x)-\Phi_{\alpha}(x)\right|=0 .
$$

Note that

$$
\frac{1}{\log N} \sum_{n=1}^{N} \frac{1}{n} f\left(\frac{M_{n}}{a_{n}}\right)=\frac{K(N)}{\log N} \int_{0}^{\infty} f(x) d F_{N}=\frac{K(N)}{\log N} \mathbb{E} f\left(Z_{N}\right) .
$$

By the definition of $\Omega$ we know that $\left\{f(Z), f\left(Z_{N}\right), N \geq 1\right\}$ is uniformly integrable. Thus by Lemma 1

$$
\lim _{N \rightarrow \infty} \frac{1}{\log N} \sum_{n=1}^{N} \frac{1}{n} f\left(\frac{M_{n}}{a_{n}}\right)=\int_{0}^{\infty} f(x) \Phi_{\alpha}(d x) .
$$

This proves (1.5).

ProOF (of Theorem 2). Put $Y_{j}=1 /\left(x_{F}-X_{j}\right)$ for $j \geq 1$. Then $\max _{1 \leq j \leq n} Y_{j}=$ $1 /\left(x_{F}-M_{n}\right)$ and

$$
\frac{\max _{1 \leq j \leq n} Y_{j}}{a_{n}^{-1}} \stackrel{d}{\rightarrow} \Phi_{\alpha}
$$

Put $f(x)=g\left(-x^{-1}\right)$ for $x>0$. Then (1.4) is satisfied because of (1.7). Using Theorem 1 we have (1.8). 
PROOF (of Theorem 3). Note that (1.1) implies

$$
\lim _{t \rightarrow \infty} \frac{U(t x)-U(t)}{U(t e)-U(t)}=\log x \quad \text { for all } x>0
$$

(see de Haan [5, Theorem 2.4.1]), using the known inequality for $\Pi$-function (see Geluk and de Haan [4, Proposition 1.19.4]), for every $\epsilon>0$, there exist $C>0$ and $t_{2}>0$ such that

$$
\left|\frac{U(t x)-U(t)}{U(t e)-U(t)}\right| \leq C\left(x^{\epsilon}+x^{-\epsilon}\right)
$$

for all $t \geq t_{2}$ and $t x \geq t_{2}$. Following the lines of proof of Theorem 1, we have (1.11).

\section{Acknowledgements}

We thank associate editor and a referee for their constructive comments and suggestions on the earlier versions of the paper. The work by Y. Qi was supported by the Netherlands Foundation for Mathematics SMC with a grant from the Netherlands Organization for Scientific Research NWO. S. Cheng and Y. Qi would like to thank Prof. L. de Haan for his hospitality and for inviting them to do research at the Erasmus University Rotterdam.

\section{References}

[1] G. A. Brosamler, 'An almost everywhere central limit theorem', Math. Proc. Camb. Philos. Soc. 104 (1988), 561-574.

[2] S. Cheng, L. Peng and Y. Qi, 'Almost sure convergence in extreme value theory', Math. Nachr. 190 (1998), 43-50.

[3] Y. S. Chow and H. Teicher, Probability theory (Springer, New York, 1988).

[4] J. Geluk and L. de Haan, Regular variation, extensions and Tauberian theorems (CWI Tract 40, Amsterdam, 1987).

[5] L. de Haan, On regular variation and its application to the weak convergence of sample extremes (CWI Tract 32, Amsterdam, 1970).

[6] M. T. Lacey and W. Philipp, 'A note on the almost sure central limit theorem', Statist. Probab. Lett. 9 (1990), 201-205.

[7] S. I. Resnick, Extreme values, regular variation, and point processes (Springer, New York, 1987).

[8] P. Schatte, 'On strong versions of the central limit theorem', Math. Nachr. 137 (1988), 249-256.

[9] — 'On the almost sure convergence of subsequences in the central limit theorem', Statistics 20 (1989), 593-605.

[10] _ - 'Two remarks on the almost sure central limit theorem', Math. Nachr. 154 (1991), 225-229. 
[11] _ 'On the central limit theorem with almost sure convergence', Probab. Math. Statist. 11 (1991), 237-246.

Department of Probability and Statistics

Peking University

Beijing, 100871

P. R. China

e-mail: shcheng@pku.edu.cn

University of Georgia

Department of Statistics

220 Statistics Building

Athens, Georgia

USA

e-mail: yqi@stat.uga.edu
Center for Mathematics and its Applications Australian National University Canberra, ACT 0200 Australia e-mail: liang.peng@maths.anu.edu.au 